\title{
Caracterización de la población con anemia en el embarazo y su asociación con la morbimortalidad perinatal
}

\author{
Martha Liliana Pérez ${ }^{1}$; María del Mar Peralta A²; Yesica Fernanda Villalba C²; Silvia Virginia Vanegas T²; Juan Diego Rivera $\mathbf{M}^{2}$; \\ Juan Diego Galindo $D^{2}$; Juan José Rubio A². \\ 1: Médico especialista en ginecología y obstetricia de la Universidad de Antioquia, ginecóloga del Hospital universitario Hernando Moncaleano \\ Perdomo; 2: Médico General - Universidad Surcolombiana.
}

Fecha de recepción:08/04/2018 Fecha de corrección: 15/11/2019

Fecha de aprobación: 20/01/2019

Fecha de publicación:30/06/2019

\section{Resumen}

Introducción: la anemia es el trastorno nutricional más frecuente en el mundo y en el embarazo constituye un riesgo para la madre, el feto o recién nacido, desencadenando complicaciones como IVU, aborto, RCIU, parto prematuro, preeclampsia, bajo peso al nacer, RPM, etc.

Objetivo: Determinar los efectos de la anemia en el embarazo y el desenlace perinatal de las pacientes atendidas en el Hospital Universitario Hernando Moncaleano de Neiva en el periodo de junio de 2012 a junio de 2016.

Metodología: Se realizó un estudio observacional, descriptivo, retrospectivo, en gestantes atendidas en consulta de alto riesgo obstétrico durante el periodo e institución mencionados, con cruce de variables para determinar la relación entre la gravedad de la anemia y sus complicaciones mediante el cálculo del Chi-cuadrado

Resultados: De 1.493 pacientes, 101 mujeres embarazadas entre los 16 a 34 años fueron incluidas en el estudio. La prevalencia de anemia fue de $32.01 \%$ y las complicaciones identificadas fueron: Infección de vías urinarias $(8,91 \%)$, restricción de crecimiento intrauterino $(7,9 \%)$, bajo peso al nacer $(6,9 \%)$, preeclampsia $(4,95 \%)$, parto prematuro $(3,96 \%)$, ruptura prematura de membranas $(3,03 \%)$ y un solo caso de aborto. El 10,89\% de los recién nacidos requirieron hospitalización, de los cuales 2 presentaron hipoglicemia y 5 ictericia neonatal.

Conclusiones: La anemia es una patología subdiagnosticada que afecta a mujeres entre los 16 y 30 años cuyas complicaciones como bajo peso al nacer, restricción de crecimiento intrauterino e ictericia neonatal resultan dependientes de la gravedad de la anemia.

Palabras clave: Embarazo; Nutrición; Anemia.

Copyright (C) Facultad de Ciencias de la Salud de la Universidad Tecnológica de Pereira. 1995-2018. Todos los derechos reservados.
Characterization of pregnant population with anemia and asociation with perinatal morbimortality:

\begin{abstract}
Introduction: Anemia is the most frequent nutritional disorder in the world and in pregnancy it is a risk for the mother, the fetus or newborn, triggering complications such as IVU, abortion, IUGR, preterm birth, preeclampsia, low birth weight, RPM, etc. Objective: To determine the effects of anemia on pregnancy and the perinatal outcome of the patients treated at the Hernando Moncaleano Hospital in Neiva from June 2012 to June 2016. Methodology: An observational, descriptive, retrospective study, In pregnant women attended at a high obstetrical risk visit during the period and institution mentioned, with crossvariables to determine the relationship between the severity of the anemia and its complications by the Chi-square calculation Results: Of 1,493 patients, 101 pregnant women Between the ages of 16 and 34 were included in the study. The prevalence of anemia was $32.01 \%$ and the complications identified were: Urinary tract infection $(8.91 \%)$, intrauterine growth restriction $(7.9 \%)$, low birth weight $(6.9 \%)$, preeclampsia, $95 \%)$, preterm delivery $(3.96 \%)$, premature rupture of membranes $(3.03 \%)$ and a single case of abortion. $10.89 \%$ of the newborns required hospitalization, of which 2 had hypoglycemia and 5 neonatal jaundice. Conclusions: Anemia is an underdiagnosed pathology that affects women between 16 and 30 whose complications such as low birth weight, intrauterine growth restriction and neonatal jaundice are dependent on the severity of the anemia.
\end{abstract}

Keywords: Pregnancy; Nutrition; Anemia

Copyright (C) Facultad de Ciencias de la Salud de la Universidad Tecnológica de Pereira. 1995-2018. Todos los derechos reservados.

\section{Introducción}

La OMS define la anemia gestacional como una hemoglobina menor o igual a $11 \mathrm{~g} / \mathrm{dl}$ y su principal causa es la mal nutrición. La deficiencia de hierro es la principal carencia nutricional en el mundo [1,2]. Además, es la principal causa de anemia en el embarazo. Esta etiología es de mayor prevalencia en los países del tercer mundo o en vía de desarrollo, dentro de los cuales se encuentra Colombia. Es importante destacar que fue reconocida como un problema de salud pública por la Organización Mundial de la Salud desde 1992 por lo que ha recomendado siempre la 
suplementación con hierro en países de bajos ingresos e incluso en los de altos ingresos. $[3,4]$

En cuanto a estadísticas internacionales realizadas por la Organización mundial de la salud, se encontró una prevalencia de anemia en mujeres embarazadas de $41,8 \%$, elevándose hasta superar la mitad de las gestantes en los países del tercer mundo.

En Colombia, de acuerdo con los reportes de las recientes Encuestas Sobre la Situación Nutricional (ENSIN), se dictamina que el 7,6\% de las mujeres en edad fértil, presentan anemia representando, según la OMS un problema de gravedad moderada indicando además que esta patología se encuentra subdiagnosticada. En el contexto internacional la deficiencia de hierro se posiciona como la causa más común, ocupando un porcentaje cercano al $75 \%$ debido a las condiciones sociales adversas, al incumplimiento de las políticas de salud pública para prevenirla y a la falta de diagnóstico durante el embarazo [5].

A lo largo de la gestación, el desarrollo del feto es completamente dependiente de los requerimientos nutricionales de su madre. Cada embarazo requiere al menos $300 \mathrm{mg}$ de hierro tomado de las reservas hepáticas de la madre [6], e incluso otros autores consideran hasta $500 \mathrm{mg}$. Esta consideración es importante cuando se sugiere que el $20 \%$ de las mujeres tienen esa reserva, y hasta un $40 \%$ en todo el mundo no tienen reservas de hierro en absoluto [7].

La anemia en el embarazo se relaciona con disminución del volumen eritrocitario, sobre un aumento del volumen plasmático materno, con la consecuente disminución de la perfusión tisular y función placentaria inadecuada, situación que puede resultar en aborto o restricción del crecimiento fetal, por tal motivo esta patología se ha asociado con cinco complicaciones obstétricas frecuentes como aborto, ruptura prematura de membranas, parto prematuro, oligohidramnios y bajo peso al nacer [8].

La gestante con anemia tiene mayor predisposición a las infecciones [9]. Es así como en las embarazadas anémicas, la infección urinaria ocurre con mayor frecuencia que en las no anémicas [10]. De igual manera la infección y dehiscencia de la herida quirúrgica suelen ser más frecuentes en las embarazadas con anemia [11]. Los trastornos hipertensivos en el embarazo también son más frecuentes en este grupo [12]; así como el incremento de las complicaciones hemorrágicas en el puerperio [13]. Las gestantes con deficiencia de hierro tienen dos veces más riesgo de presentar un parto prematuro, y el triple de tener un feto con bajo peso [14].

El tratamiento de la anemia por deficiencia de hierro en el embarazo principal causa de esta patología- se logra mediante la suplementación en la dieta y además la toma oportuna de hierro y folato, a fin de prevenir la evolución a una anemia más grave. La American Congress of Obstetricians and Gynecologists (ACOG) recomienda actualmente establecer un esquema terapéutico de 160 a $200 \mathrm{mg} /$ día de hierro ferroso elemental, con lo cual se espera un aumento de $\mathrm{Hb}$ de $1 \mathrm{~g} /$ dl después de 14 días de terapia, permitiendo evidenciar además de manera oportuna si el tratamiento es funcional y por tanto conocer si el diagnóstico fue certero. Según el reporte de CHERG (Child Health Epidemiology Reference Group) acerca de deficiencia de hierro, la morbimortalidad disminuye significativamente por cada gramo de hemoglobina que se incremente [15].
Debido al aumento del riesgo de anemia con el progreso del embarazo y siendo un problema de salud pública en departamentos con una alta tasa de embarazos con riesgo social elevado -debido a pobreza y otras condiciones adversas- como el nuestro, donde las dietas son pobres en hierro y por lo tanto las reservas en las gestantes son escasas, es menester caracterizar nuestra población y determinar la morbimortalidad perinatal debido a esta patología para así saber con certeza la funcionalidad de la aplicación de estrategias para prevenirla y predecir lo que nos deparan los próximos años respecto a la salud materno-fetal.

\section{Materiales y métodos.}

Se realizó un estudio observacional, descriptivo, retrospectivo, longitudinal, en gestantes atendidas en la consulta de alto riesgo obstétrico en el Hospital Universitario Hernando Moncaleano Perdomo de la ciudad de Neiva durante el período de junio del 2012 a junio del 2016, con muestra no probabilística, no aleatoria, seleccionada a conveniencia de los investigadores. A través del archivo digital Índigo Cristal del hospital, se obtuvo una cantidad de 1493 registros clínicos de mujeres embarazadas atendidas en consulta de alto riesgo obstétrico durante este periodo, de las cuales fueron seleccionadas solamente 478 pacientes con diagnóstico de anemia y a su vez se incluyeron para el estudio 101 pacientes con base en los siguientes criterios de inclusión: Mujeres embarazadas con hemoglobina menor a $11 \mathrm{~g} / \mathrm{dl}$, cuyo embarazo fuese único, con una historia clínica completa.

Los criterios de exclusión fueron los siguientes: Mujeres con antecedente de parto pretérmino, hipertensión crónica, preeclampsia, gestante VIH positivo, RCIU, muerte perinatal o neonatal, ruptura prematura de membranas o algún antecedente patológico personal.

Tras seleccionar las 101 pacientes, se procedió a tabular la información recolectada en hojas de cálculo del programa Excel de Microsoft, tras lo cual se realizó el análisis estadístico de las variables establecidas en el instrumento mediante el software estadístico Epi Info ${ }^{\mathrm{TM}}$ para calcular la frecuencia y los porcentajes, además se generó un cruce de variables que permitió correlacionar la gravedad de la anemia con cada una de las complicaciones estudiadas, determinando si existía o no significancia estadística mediante el cálculo del Chicuadrado y estableciendo si las hipótesis planteadas eran o no válidas según la prueba no paramétrica de Pearson.

\section{Resultados.}

En total, 101 pacientes cumplieron con los parámetros de selección de nuestro estudio. De ellas las mujeres entre los 16 a los 34 años constituyeron el grupo etario más grande, solo se encontró una paciente con más de 34 años y 5 pacientes menores de 16 años.

La prevalencia de anemia fue de $32.01 \%$ teniendo en cuenta el total de 1493 pacientes que asistieron a consulta de alto riesgo obstétrico durante junio de 2012 y junio de 2016, de las cuales 478 tenían como diagnóstico anemia durante el embarazo, este resultado es debido a la aplicación de los criterios como la exclusión de las pacientes quienes presentaban patologías asociadas igualmente quienes no presentaron parto en nuestra instituciones lo cual generó una disminución de la muestra estudiada. 


\begin{tabular}{|c|c|c|c|}
\hline Variable & $\mathbf{N}$ & $\%$ & IC $95 \%$ \\
\hline \multicolumn{4}{|l|}{ Edad } \\
\hline$<16$ Años & 5 & 4,9 & \\
\hline $16-35$ Años & 95 & 94 & \\
\hline > 35 Años & 1 & 0,99 & \\
\hline \multicolumn{4}{|l|}{ Gestaciones } \\
\hline $1-4$ & 93 & 92,08 & $84,99-96,5$ \\
\hline$>4$ & 8 & 7,92 & $3,48-15,01$ \\
\hline \multicolumn{4}{|l|}{ Partos } \\
\hline 0 & 42 & 41,58 & $31,86-51,82$ \\
\hline $1-4$ & 58 & 57,43 & $47,19-67,21$ \\
\hline$>4$ & 1 & 0,99 & $0,03-5,39$ \\
\hline \multicolumn{4}{|l|}{ Abortos } \\
\hline 0 & 85 & 84,16 & $75,5-90,6$ \\
\hline $1-4$ & 16 & 15,84 & $9,3-24,45$ \\
\hline \multicolumn{4}{|l|}{ Cesáreas } \\
\hline 0 & 59 & 58,42 & $48,1-68,1$ \\
\hline $1-4$ & 42 & 41,58 & $31,8-51,8$ \\
\hline \multicolumn{4}{|l|}{ Nacidos Muertos } \\
\hline 0 & 99 & 98,02 & $93-99,7$ \\
\hline $1-4$ & 2 & 1,98 & $0,2-6,9$ \\
\hline
\end{tabular}

Tabla 1 Antecedentes ginecológicos de las pacientes con anemia atendidas en el Hospital Universitario Hernando Moncaleano de Neiva 2012 2016

Con respecto a los antecedentes ginecológicos, el $92 \%$ de las pacientes no habían cursado con más de 4 embarazos y solo un $8 \%$ aproximado habían tenido más de 4 embarazos. 16 pacientes tenían antecedentes de aborto y 2 antecedentes de recién nacidos muertos, con causa no especificada en el registro clínico. (Tabla 1).

\begin{tabular}{cccc}
\hline Variable & N & I & I5\% \\
\hline Edad gestacional al momento del parto (semanas) & & 9 & $4,2-16,4$ \\
$<37$ & 9 & 87 & $78,8-92,89$ \\
$37-41$ & 87 & 4 & $1,1-9.93$ \\
$>41$ & 4 & 65,35 & $55,23-74,54$ \\
Vía del parto & 66 & 34,65 & $25,46-44,77$ \\
Parto vaginal & 35 & & \\
Cesárea & & 35 & \\
\hline
\end{tabular}

Tabla 2 Momento y vía del parto en las pacientes con anemia atendidas en el Hospital Universitario Hernando Moncaleano de Neiva 2012 - 2016

El parto vaginal fue la principal vía de parto en las mujeres con diagnóstico de anemia gestacional (65,35\%) y también el principal antecedente ginecológico de vía de parto entre las pacientes.

Respecto a la edad gestacional al momento del parto, el $87 \%$ de las mujeres llevaron a término su gestación sin sobrepasar las 41 semanas, mientras un $9 \%$ de las pacientes tuvieron parto antes de la semana 37 , haciendo claridad que se presentó un aborto. (Tabla 2)

Con base en la hemoglobina documentada antes de la semana 20, el 52,48\% de las pacientes presentaron anemia leve, $44,5 \%$ anemia moderada y $2,97 \%$ anemia severa; adicionalmente; el 63\% tenía registro clínico de haber recibido tratamiento con hierro y 5,94\% ${ }^{[15]}$, recibieron transfusión sanguínea de aquellas pacientes que fueron transfundidas 3 tenían anemia severa, 2 pacientes con anemia modera fueron transfundidas, 1 paciente con anemia leve fue transfundida como limitación se encuentra que no hay claridad en la historia clínica respecto a las indicación para realizar la transfusión. 


\begin{tabular}{|c|c|c|c|c|}
\hline & Variable & $\mathbf{N}$ & $\%$ & IC $95 \%$ \\
\hline \multicolumn{5}{|l|}{ Gravedad Anemia } \\
\hline & Leve $(10-11 \mathrm{~g} / \mathrm{dl})$ & 53 & 52,48 & $42,3-62,5$ \\
\hline & Moderada $(7-9,9 \mathrm{~g} / \mathrm{dl})$ & 45 & 44,55 & $34,6-54,7$ \\
\hline & Grave $(<7 \mathrm{~g} / \mathrm{dl})$ & 3 & 2,97 & $0,6-8,4$ \\
\hline \multicolumn{5}{|c|}{ Tratamiento Hierro (g) } \\
\hline & SI & 63 & 63 & $52,7-72,4$ \\
\hline & $\mathrm{NO}$ & 36 & 36 & $26,6-46,2$ \\
\hline \multicolumn{5}{|l|}{ Transfusiones } \\
\hline & SI & 6 & 5,94 & $2,21-12,48$ \\
\hline & NO & 95 & 94,06 & $87,52-97,79$ \\
\hline \multicolumn{5}{|l|}{ HB pos tratamiento } \\
\hline & $9-10 \mathrm{~g} / \mathrm{dl}$ & 11 & 10,8 & $5,5-18,6$ \\
\hline & $10.1-11$ & 23 & 22,7 & $15-32$ \\
\hline & $11.1-12$ & 18 & 17,8 & $10,9-26,7$ \\
\hline & $>12$ & 16 & 15,8 & $9,3-24,4$ \\
\hline & $\mathrm{SD} / \mathrm{NA}^{*}$ & 33 & 32,6 & $23,6-42,7$ \\
\hline \multicolumn{5}{|l|}{ HB Parto } \\
\hline & $7-9.9 \mathrm{~g} / \mathrm{dl}$ & 12 & 11,8 & $6,2-19,8$ \\
\hline & $10-11 \mathrm{~g} / \mathrm{dl}$ & 40 & 39,6 & $30-49,8$ \\
\hline & $>11 \mathrm{~g} / \mathrm{dl}$ & 41 & 40,5 & $30,9-50,8$ \\
\hline & $\mathrm{SD} / \mathrm{NA}$ & 8 & 7,9 & $3,4-15$ \\
\hline *HB: hemoglobina & SD: Sin dato *NA: No aplica & & & \\
\hline
\end{tabular}

Tabla 3 Gravedad y Manejo Terapéutico de las pacientes con anemia atendidas en el Hospital Universitario Hernando Moncaleano de Neiva $2012-2016$

Hubo una mejoría en los niveles de hemoglobina comparando los registros antes de las 20 semanas y los niveles de hemoglobina posteriores al tratamiento y en el momento del parto (Tabla 3).

\begin{tabular}{|c|c|c|c|}
\hline Variable & $\mathbf{N}$ & $\%$ & IC 95\% \\
\hline \multicolumn{4}{|c|}{ Bajo peso al nacer } \\
\hline SI & 7 & 6,9 & $2,8-13,7$ \\
\hline $\mathrm{NO}$ & 94 & 93,1 & $86,2-97,1$ \\
\hline \multicolumn{4}{|l|}{ RCIU } \\
\hline SI & 8 & 7,9 & $3,8-15$ \\
\hline NO & 93 & 92 & $84,9-96,5$ \\
\hline \multicolumn{4}{|l|}{ Aborto } \\
\hline SI & 1 & 0,99 & $00,3-5,39$ \\
\hline NO & 100 & 99,01 & $94,61-99,67$ \\
\hline \multicolumn{4}{|c|}{ Parto Prematuro $(24-36,6)$} \\
\hline SI & 4 & 3,96 & $1,09-9,83$ \\
\hline NO & 97 & 96,04 & $90,17-98,91$ \\
\hline \multicolumn{4}{|c|}{ Muerte perinatal } \\
\hline SI & 0 & 0,00 & $100-100$ \\
\hline NO & 101 & 100,00 & $100-100$ \\
\hline \multicolumn{4}{|l|}{ RPM } \\
\hline SI & 3 & 3,03 & $0,62-8,44$ \\
\hline NO & 98 & 97,03 & $91,56-99,38$ \\
\hline
\end{tabular}


IVU

$\begin{array}{cccc}\text { SI } & 9 & 8,91 & 4,16-16,24 \\ \text { NO } & 92 & 91,09 & 83,76-95,84\end{array}$

Preeclampsia

$\begin{array}{cccc}\text { SI } & 5 & 4,95 & 1,63-11,18 \\ \text { NO } & 96 & 95,05 & 88,82-98,37\end{array}$

Tabla 4 Complicaciones por anemia de las pacientes atendidas en el Hospital Universitario Hernando Moncaleano de Neiva 2012 - 2016

Respecto a los resultados fetales y complicaciones asociadas, en orden de frecuencia se presentaron de la siguiente manera: Infección de vías urinarias $(8,91 \%)$, restricción de crecimiento intrauterino $(7,9 \%)$, bajo peso al nacer $(6,9 \%)$, preeclampsia $(4,95 \%)$, parto prematuro $(3,96 \%)$, ruptura prematura de membranas $(3,03 \%)$ y se presentó un solo caso de aborto. No se reportó ninguna muerte perinatal o neonatal (Tabla 4$)$.

El 10,89\% de los recién nacidos requirieron hospitalización posterior al parto, de los cuales 2 hicieron hipoglicemia neonatal y 5 ictericia neonatal. En este estudio no se encontró ninguna muerte perinatal ni muerte materna.

\begin{tabular}{|c|c|c|c|c|c|c|}
\hline COMPLICACIONES & LEVE & MODERADA & GRAVE & TOTAL & Chi $^{2}$ & P $(0.05 \%)$ \\
\hline \multirow{2}{*}{ Bajo peso al nacer } & 0 & 5 & 1 & 6 & \multirow{2}{*}{9,226} & $<0.005$ \\
\cline { 2 - 6 } & $0 \%$ & $11,10 \%$ & $33,30 \%$ & $6,10 \%$ & & \\
\hline \multirow{2}{*}{ RCIU } & 0 & 6 & 1 & 7 & \multirow{2}{*}{9,716} & $<0.005$ \\
\cline { 2 - 6 } & $0 \%$ & $13,30 \%$ & $33,30 \%$ & $7,10 \%$ & & \\
\cline { 2 - 5 } & $2 \%$ & $2,20 \%$ & $0 \%$ & $2 \%$ & & \\
\cline { 2 - 6 } Ictericia neonatal & 2 & 1 & 1 & 4 & \multirow{2}{*}{8,203} & $<0.005$ \\
\cline { 2 - 5 } & $3,90 \%$ & $2,20 \%$ & $33,30 \%$ & $4 \%$ & & \\
\hline
\end{tabular}

Tabla 5 Complicaciones de acuerdo al grado de anemia de las pacientes atendidas en el Hospital Universitario Hernando Moncaleano de Neiva 2012 - 2016

Al analizar las complicaciones encontradas en la población con anemia diagnosticada durante el embarazo y compararla respecto a la gravedad se evidenciaron a través de la prueba de chi cuadrado tres complicaciones dependientes de la severidad: bajo peso al nacer, restricción de crecimiento intrauterino e ictericia neonatal (Tabla 5).

\section{Discusión.}

La prevalencia encontrada en nuestra población no difiere significativamente de la encontrada en la literatura mundial (41,8\%), en especial de las mujeres latinas $(30,1 \%)$ [16]. Así mismo las complicaciones encontradas se presentaron con un orden de frecuencia análogo, sin embargo, no se evidenció una relación clara con la anemia.

De los diferentes estudios encontrados en la literatura, la edad promedio en todos varió entre los 24,6 años a los 29,3 años, lo que indicaría que existe una concordancia con nuestro estudio en donde el principal grupo etario de mujeres embarazadas con diagnóstico de anemia se encontraban entre los 16 y 30 años, representando un factor positivo ya que en muy pequeña proporción confluyeron adolescencia y embarazo que demandan mayores necesidades de hierro. Ahora bien, una diferencia identificada fue la ausencia de una relación directamente proporcional entre el número de partos y la anemia gestacional. Agregado, hubo más partos vaginales que cesáreas a pesar del grado de anemia como en la mayoría de los estudios $[16,17]$.

La gravedad de la anemia fue similar entre la literatura mundial, regional y nuestra investigación, encontrando un mayor porcentaje de anemia leve (siempre dentro del rango del 50 al 60\% e incluso llegando hasta el 70\% de los casos en investigaciones de países desarrollados) seguida por la anemia moderada (entre el 40 y el 50\%) y con prácticamente ningún caso de anemia grave; sin embargo, nos hallamos por encima de la estadística si analizamos las demás investigaciones donde la anemia grave nunca llegó a cifras como las encontradas localmente.[16,17,18]

Al igual que en los demás estudios relacionados con el tema, no encontramos una clara relación entre la anemia y las posibles complicaciones derivadas de ella posiblemente a la limitación de la muestra de 101 pacientes principalmente por registros clínicos incompletos, atención del parto extrainstitucional y sub diagnóstico; llevando a que la prevalencia de patologías como el bajo peso al nacer, la restricción del crecimiento 
intrauterino, el parto pretérmino, la ruptura prematura de membranas o la hospitalización postparto, no fuera mayor que en los embarazos de grávidas sin el diagnóstico de anemia gestacional.

La complicación que más se presentó en frecuencia fue la infección de vías urinarias, en donde se debe tener en cuenta que aproximadamente el 2-7\% de las embarazadas presenta IVU en algún momento de la gestación, siendo más frecuente en multíparas, en medio socioeconómico bajo y de acuerdo con la edad (a mayor edad, mayor predisposición a este tipo de infecciones) [1], sin embargo, no hubo relación con la gravedad de la anemia.

Las complicaciones como bajo peso al nacer, restricción del crecimiento intrauterino e ictericia neonatal presentaron una dependencia de la gravedad de la anemia. Señalando que los porcentajes de las complicaciones mencionadas fueron similares a las estadísticas de la literatura e incluso inferiores, teniendo en cuenta, por ejemplo, el metaanálisis realizado por Mizanur Et al. publicado en el 2016, en donde encontraron un aumento significativo del riesgo de bajo peso al nacer (RR:1,31), parto pretérmino (RR:1.63), mortalidad perinatal (RR: 1.51) y mortalidad neonatal (RR:2.72). Igualmente, los resultados de nuestro estudio son concordantes con otros estudios observacionales realizados en países de bajos y medianos ingresos, en donde el $12 \%$ de los casos de bajo peso al nacer, el 19\% de los partos prematuros, y el $18 \%$ de la mortalidad perinatal han sido atribuidos a la anemia materna [19, 20, 21, 22, 23]. En contraste con estos mismos, no se presentó ningún caso de mortalidad materna ni perinatal.

Es importante recalcar que la anemia como patología no está siendo incluida dentro de los diagnósticos de las pacientes a pesar del reporte paraclínico de la misma aumentando el número de casos subdiagnosticados.

De acuerdo con las pacientes que fueron trasfundidas en nuestra investigación, parece no existir un consenso universal para definir conducta de transfusión en la población con anemia en el Hospital Universitario Hernando Moncaleano, en donde no se tuvo en cuenta la gravedad de la anemia sin causa justificada en los registros clínicos.

Finalmente, deben tenerse en cuenta otros factores como estatus socioeconómico, nivel de atención médica y estado nutricional que pueden influir en la morbimortalidad y se recomienda realizar estudios de carácter prospectico que evalúen la duración de la anemia más que su severidad para demostrar el efecto de la intervención temprana y el tratamiento de la anemia en los resultados materno-infantiles.

\section{Conflicto de intereses.}

Los autores declaran no tener conflictos de interés.

\section{Referencias.}

1. Stoltzfus R, Dreyfuss M. Guidelines for the use of iron supplements to prevent and treat iron deficiency anaemia. ILSI Press, 1998.

2. World Health Organization. Micronutrient deficiencies: iron deficiency anaemia. www.who. int/nutrition/topics/ ida/en/
3. Institute of Medicine. Iron deficiency anemia: guidelines for prevention, detection and management among U.S. children and women of childbearing age. National Academy Press, 1993.

4. Centers for Disease Control and Prevention. Recommendations to prevent and control iron deficiency in the United States. MMWR Recomm Rep 1998;47(RR3):1-29.

5. Hoz FED La, Santiago LO. Anemia en el embarazo, un problema de salud que puede prevenirse. Rev Médicas Uis [Internet]. 2014;26(3):45-50.

6. Bothwell $\mathrm{TH}$ (2000) Iron requirements in pregnancy and strategies to meet them. Am J Clin Nutr 72, 257S-264S

7. Baker RD \& Greer FR (2010) Diagnosis and prevention of iron deficiency and iron deficiency anemia in infants and young children ( $0-3$ years of age). Pediatrics 126,1040 1050 .

8. Milman N. Iron and pregnancy-a delicate balance.Ann Hematol.2006;85(9):559-65

9. http://www.accessdata.fda.gov/drugsatfda_docs/ label/2009/022180lbl.pdf

10. Protocolo de Vigilancia Epidemiológica para Malaria. Actualizado por Luis Armando Galeano Marín, médico especialista, el 31 de julio de 2009. Direccion Seccional De Salud Y Protección Social De Antioquia.

11. Pels S, Paidas M. Microangiopathic disorders in pregnancy. Hematol Oncol Clin North Am.2011;25(2):311-22.

12. Steer P. Maternal hemoglobin concentration and birth weight.Am J Clin Nutr.2000;71(5 Suppl):1285-7

13. SIfakis A, Pharmakides G. Anemia in pregnancy. Annals of the New York Academy of Sciences 2000; 900:125-136

14. Lee A, Okam MM. Anemia in pregnancy.Hematol Oncol Clin North Am.2011;25(2):241-59.

15. Murray-Kolb L: Maternal Mortality, Child Mortality, Perinatal Mortality, Child Cognition, and Estimates of Prevalence of Anemia due to Iron Deficiency. In: CHERG. 2012.

16. Espitia De La Hoz, F., \& Orozco Santiago, L. (2013). Anemia en el embarazo, un problema de salud que puede prevenirse. Medicas UIS, 26(3), 45-50.

17. Tamar Tzur1, Adi Y. Weintraub Can anemia in the first trimester predict obstetrical complications later in pregnancy? ISSN 1476-7058 print/ISSN 1476-4954

18. MSc. Irania clara, Suárez san gil; MSc. Villazán cristina, Characterization of anemia during pregnancy and some 
associated risk factors in pregnant women at Regla municipality. Rev Cubana Med Gen Integr vol.30 no.1 Ciudad de La Habana ene.mar. 2014 ISSN 0864-2125.

19. Solange Augusta de Sá, Erica Willner, Anemia in pregnancy: impact on weight and in the development of anemia in newborn, Nutr Hosp. 2015;32(5):2071-2079 ISSN 0212-1611

20. Rahman, M. M., Abe, S. K., Rahman, M. S., Kanda, M., Narita, S., Bilano, V., ... \& Shibuya, K. (2016). Maternal anemia and risk of adverse birth and health outcomes in low-and middle-income countries: systematic review and meta-analysis. The American journal of clinical nutrition, 103(2), 495-504.

21. Pena-Rosas JP, Viteri FE. Effects and safety of preventive oral iron or iron+folic acid supplementation for women during pregnancy. Cochrane Database Syst Rev 2009;(4):CD004736

22. Pena-Rosas JP, Viteri FE. Effects and safety of preventive oral iron or iron+folic acid supplementation for women during pregnancy. Cochrane Database Syst Rev 2009;(4):CD004736

23. Imdad A, Bhutta ZA. Routine iron/folate supplementation during pregnancy: effect on maternal anaemia and birth outcomes. Paediatr Perinat Epidemiol 2012;26(suppl 1):168-77 